\title{
Delta-Function Potential with a Complex Coupling
}

\author{
Ali Mostafazadeh \\ Department of Mathematics, Koç University, \\ 34450 Sariyer, Istanbul, Turkey \\ amostafazadeh@ku.edu.tr
}

\begin{abstract}
We explore the Hamiltonian operator $H=-\frac{d^{2}}{d x^{2}}+z \delta(x)$ where $x \in \mathbb{R}, \delta(x)$ is the Dirac delta function, and $z$ is an arbitrary complex coupling constant. For a purely imaginary $z, H$ has a spectral singularity at $E=-z^{2} / 4 \in \mathbb{R}^{+}$. For $\Re(z)<0, H$ has an eigenvalue at $E=-z^{2} / 4$. For the case that $\Re(z)>0, H$ has a real, positive, continuous spectrum that is free from spectral singularities. For this latter case, we construct an associated biorthonormal system and use it to perform a perturbative calculation of a positive-definite inner product that renders $H$ self-adjoint. This allows us to address the intriguing question of the nonlocal aspects of the equivalent Hermitian Hamiltonian for the system. In particular, we compute the energy expectation values for various Gaussian wave packets to show that the non-Hermiticity effect diminishes rapidly outside an effective interaction region.
\end{abstract}

PACS number: 03.65.-w

Keywords: Complex potential, delta function, pseudo-Hermitian, inner product, metric operator, spectral singularity, $\mathcal{P} \mathcal{T}$-symmetry 


\section{Introduction}

The observation that a complex potential can define a consistent unitary quantum system has recently led to a considerable research activity. This is mostly focused on the study of the complexvalued $\mathcal{P} \mathcal{T}$-symmetric potentials $v$ for which the Hamiltonian

$$
H=\frac{p^{2}}{2 m}+v(x)
$$

has a real discrete spectrum. In general the eigenvalue problem for $H$ is defined along an appropriate contour $\Gamma$ in the complex plane with suitable boundary conditions at infinity [1]. This allows one to identify $H$ with a densely defined and generally non-self-adjoint operator acting in a separable Hilbert space $\mathcal{H}$, with the typical choice for $\mathcal{H}$ being $L^{2}(\Gamma)$.

The problem of whether and how one can formulate a consistent quantum system having $H$ as its Hamiltonian has found a satisfactory solution within the context of pseudo-Hermitian quantum mechanics [2, 3, 4, 5]. It turns out that $H$ must be diagonalizable. In particular, there must exist a complete basis of $\mathcal{H}$ consisting of eigenvectors of $H$. This is a physical requirement of the standard quantum measurement postulate [2, 6]. For a diagonalizable Hamiltonian with a discrete spectrum, the reality of the spectrum is equivalent to the existence of a positive-definite inner product $\langle\cdot, \cdot\rangle_{+}$that renders $H$ self-adjoint [7, 8]. The latter is a necessary and sufficient condition for the existence of an equivalent Hermitian Hamiltonian $h$ that acts in $\mathcal{H}$, [7, 9]. These observations lead to the realization that the physical system under investigation may be equally well described by a Hermitian Hamiltonian within the framework of the conventional quantum mechanics [10, 11, 2].

The above discussion also applies to complex potentials that are not $\mathcal{P} \mathcal{T}$-symmetric. The purpose of this paper is to study in detail one of the simplest (though highly nontrivial) examples of complex potentials that happens not to be $\mathcal{P} \mathcal{T}$-symmetric, namely the delta-function potential with a complex coupling:

$$
v(x)=\zeta \delta(x), \quad \zeta \in \mathbb{C} .
$$

We wish to explore the possibility of defining a unitary quantum system based on the standard Hamiltonian (11) and the potential (2). The reference Hilbert space [2] is given by $\mathcal{H}=L^{2}(\mathbb{R})$ and it is not difficult to see that the spectrum of the Hamiltonian fails to be discrete. Because of the presence of the continuous part of the spectrum the results reported in [12, 7] may not hold. Nevertheless, they provide some useful guiding principles that we will follow. Specifically, we will attempt to construct a biorthonormal system whenever possible and use it to define an appropriate positive-definite inner product that renders $H$ self-adjoint. An example of a successful application of this strategy is the scattering potential [5]

$$
v(x)=\left\{\begin{array}{ccc}
-i \lambda \operatorname{sign}(x) & \text { for } & |x|<\frac{L}{2} \\
0 & \text { for } & |x|>\frac{L}{2},
\end{array},\right.
$$


where $\lambda \in \mathbb{R}, L \in \mathbb{R}^{+}$, and

$$
\operatorname{sign}(x):=\left\{\begin{array}{ccc}
-1 & \text { for } & x<0 \\
0 & \text { for } & x=0 \\
1 & \text { for } & x>0
\end{array}\right.
$$

Note that unlike (3) , the delta-function potential (21) fails to be $\mathcal{P} \mathcal{T}$-symmetric, and as we will see, depending on the value of $\zeta$, it may lead to the presence of a spectral singularity [13].

Before starting our analysis of the properties of (2), we wish to point out that complex potentials consisting of one or more delta functions have been studied in [14 - 21] and that the issue of the emergence of spectral singularities for $\mathcal{P} \mathcal{T}$-symmetric potentials has been considered in [22].

\section{Spectral Properties and Biorthonormal Systems}

Consider the time-independent Schrödinger equation,

$$
H \psi=E \psi
$$

subject to bounded boundary conditions at $x= \pm \infty$. Substituting (11) and (2) in (44) and introducing the dimensionless quantities:

$$
\mathrm{x}:=\frac{x}{\ell}, \quad z:=\frac{2 m \ell \zeta}{\hbar^{2}}, \quad \mathrm{E}:=\frac{2 m \ell^{2} E}{\hbar^{2}},
$$

where $\ell$ is an arbitrary length scale, we can express (44) in the form

$$
-\psi^{\prime \prime}(\mathrm{x})+z \delta(\mathrm{x}) \psi(\mathrm{x})=\mathrm{E} \psi(\mathrm{x})
$$

Clearly E belongs to the spectrum of the dimensionless Hamiltonian

$$
\mathrm{H}:=\frac{2 m \ell^{2}}{\hbar^{2}} H=-\frac{d^{2}}{d \mathrm{x}^{2}}+z \delta(\mathrm{x}) .
$$

The solution of (6) has the form

$$
\psi(\mathrm{x})=\psi_{k}(\mathrm{x}):= \begin{cases}A_{-} e^{i k \mathrm{x}}+B_{-} e^{-i k \mathrm{x}} & \text { for } \mathrm{x}<0 \\ A_{+} e^{i k \mathrm{x}}+B_{+} e^{-i k \mathrm{x}} & \text { for } \mathrm{x} \geq 0\end{cases}
$$

where $k:=\sqrt{\mathrm{E}}$, the coefficients $A_{-}, B_{-} \in \mathbb{C}$ are arbitrary but not both vanishing, i.e., $\left|A_{-}\right|^{2}+$ $\left|B_{-}\right|^{2} \neq 0$, and

$$
A_{+}=\left(1-\frac{i z}{2 k}\right) A_{-}-\frac{i z}{2 k} B_{-}, \quad B_{+}=\frac{i z}{2 k} A_{-}+\left(1+\frac{i z}{2 k}\right) B_{-} .
$$

A straightforward implication of (8) and (9) is that whenever $\Re(z)<0$ there is a solution $\psi_{k}$ with $k=i z / 2$ that belongs to $L^{2}(\mathbb{R})$, i.e., the spectrum consists of the obvious real, nonnegative, continuous part and a single eigenvalue: $\mathrm{E}:=-z^{2} / 4$. 
The presence of a pair $\left(A_{-}, B_{-}\right)$of arbitrary constants in the expression for the eigenfunctions $\psi_{k}$ is an indication that the energy levels are doubly degenerate. The application of the program of pseudo-Hermitian quantum mechanics [2] requires the construction of a complete biorthonormal system consisting of the eigenfunctions of $\mathrm{H}$ and $\mathrm{H}^{\dagger}$. The first step in this direction is to make a convenient choice for basis eigenfunctions within each degeneracy subspace. It is most convenient to choose one of these eigenfunctions reflectionless [23] - 25]. This would allow one to express this eigenfunction using a formula that is valid for both $\mathrm{x}<0$ and $\mathrm{x} \geq 0$. It is not difficult to see that $\sin (k \mathrm{x})$ is such a reflectionless eigenfunction. Moreover, the fact that $\sin (k \mathrm{x})$ is an odd function suggests to choose the second basis eigenfunction to be even 1 As we will see below, this choice simplifies the imposition of the biorthogonality conditions considerably.

Denoting the eigenfunctions by $\tilde{\psi}_{a}^{k}$, with $a=1,2$ being the degeneracy label, we set

$$
\begin{aligned}
\tilde{\psi}_{1}^{k}(\mathrm{x}) & :=\frac{1}{\sqrt{\pi}} \sin (k \mathrm{x}) \\
\tilde{\psi}_{2}^{k}(\mathrm{x}) & :=\frac{1}{\sqrt{\pi}}\left[\cos (k \mathrm{x})+\frac{z}{2 k} \sin (k \mathrm{x}) \operatorname{sign}(\mathrm{x})\right] .
\end{aligned}
$$

We can construct the following eigenfunctions of $\mathrm{H}^{\dagger}$ by replacing $z$ by $z^{*}$ in the above formulas.

$$
\begin{aligned}
\tilde{\phi}_{1}^{k}(\mathrm{x}) & :=\frac{1}{\sqrt{\pi}} \sin (k \mathrm{x}) \\
\tilde{\phi}_{2}^{k}(\mathrm{x}) & :=\frac{1}{\sqrt{\pi}}\left[\cos (k \mathrm{x})+\frac{z^{*}}{k} \sin (k \mathrm{x}) \operatorname{sign}(\mathrm{x})\right] .
\end{aligned}
$$

Next, we wish to check the validity of the biorthonormality relation for the system $\left\{\tilde{\psi}_{a}^{k}, \tilde{\phi}_{a}^{k}\right\}$. Using the well-known integral representation of the delta function, $\delta(k)=(2 \pi)^{-1} \int_{-\infty}^{\infty} e^{i k x} d x$, the parity of the eigenfunctions, and the fact that $k+q>0$, we immediately find

$$
\left\langle\tilde{\psi}_{1}^{k} \mid \tilde{\phi}_{1}^{q}\right\rangle=\delta(k-q), \quad\left\langle\tilde{\psi}_{1}^{k} \mid \tilde{\phi}_{2}^{q}\right\rangle=\left\langle\tilde{\psi}_{2}^{k} \mid \tilde{\phi}_{1}^{q}\right\rangle=0
$$

Much more complicated is the derivation of

$$
\begin{aligned}
\left\langle\tilde{\psi}_{2}^{k} \mid \tilde{\phi}_{2}^{q}\right\rangle & =\left(1+\frac{z^{* 2}}{4 k^{2}}\right) \delta(k-q)+\pi z^{*} \delta(k) \delta(q) \\
& =\left(1+\frac{z^{* 2}}{4 k^{2}}\right) \delta(k-q), \quad \text { for } k, q>0 .
\end{aligned}
$$

As seen from (15), the system $\left\{\tilde{\psi}_{a}^{k}, \tilde{\phi}_{a}^{k}\right\}$ fails to be biorthogonal for $z= \pm 2 i k$, because $\tilde{\phi}_{2}^{k}$ is orthogonal to both $\tilde{\psi}_{1}^{q}$ and $\tilde{\psi}_{2}^{q}$ for all $q \in \mathbb{R}^{+}$. This is an indication of the presence of a spectral singularity, namely $\mathrm{E}=-z^{2} / 4$, which occurs whenever $z$ is purely imaginary 3 and is consequently embedded in the continuous spectrum of $\mathrm{H}$.

\footnotetext{
${ }^{1}$ This is possible, because the Hamiltonian is parity-invariant ( $\mathcal{P}$-symmetric).

${ }^{2}$ Here we used the identity $\delta(k)=\lim _{n \rightarrow \infty} \sin (n k) /(\pi k)$.

${ }^{3}$ This is because $k \in \mathbb{R}^{+}$and $z= \pm 2 i k$.
} 
A spectral singularity is a serious defect that rules out the operator as a viable candidate for a physical observable. We will therefore only consider non-imaginary couplings $z$. In particular, we will focus our attention on the cases where $z$ (and hence $\zeta$ ) has a positive real part $(\Re(z)>0$ ), so that there is no eigenvalue and the spectrum is $\mathbb{R}^{+} \cup\{0\}$. In this case, we can define

$$
\begin{array}{ll}
\psi_{1}^{k}(\mathrm{x}):=\frac{1}{\sqrt{\pi}} \sin (k \mathrm{x}), & \psi_{2}^{k}(\mathrm{x}):=\frac{\cos (k \mathrm{x})+\frac{z}{2 k} \sin (k \mathrm{x}) \operatorname{sign}(\mathrm{x})}{\sqrt{\pi\left(1+\frac{z^{2}}{4 k^{2}}\right)}} \\
\phi_{1}^{k}(\mathrm{x}):=\frac{1}{\sqrt{\pi}} \sin (k \mathrm{x}), & \phi_{2}^{k}(\mathrm{x}):=\frac{\cos (k \mathrm{x})+\frac{z^{*}}{2 k} \sin (k \mathrm{x}) \operatorname{sign}(\mathrm{x})}{\sqrt{\pi\left(1+\frac{z^{* 2}}{4 k^{2}}\right)}}
\end{array}
$$

which satisfy the biorthonormality condition

$$
\left\langle\psi_{a}^{k} \mid \phi_{b}^{q}\right\rangle=\delta_{a b} \delta(k-q)
$$

Clearly, this relation is invariant under the transformations

$$
\psi_{a}^{k}(\mathrm{x}) \rightarrow \psi_{a}^{\prime k}(\mathrm{x}):=N_{a}(z, k) \psi_{a}^{k}(\mathrm{x}), \quad \phi_{a}^{k}(\mathrm{x}) \rightarrow \phi_{a}^{\prime k}(\mathrm{x}):=N_{a}(z, k)^{-1 *} \phi_{a}^{k}(\mathrm{x})
$$

where $N_{a}: \mathbb{C} \times \mathbb{R}^{+} \rightarrow \mathbb{C}$ are functions that tend to 1 as $k \rightarrow \infty$ and $N_{a}(z, k)^{ \pm 1}$ do not vanish except possibly for imaginary values of $z$.

\section{Construction of a Metric Operator}

Extending the results of [12, 7] to the model under investigation, we wish to construct a positivedefinite metric operator of the form [5, 26, 27]

$$
\eta_{+}=\sum_{a=1}^{2} \int_{0}^{\infty} d k\left|\phi_{a}^{k}\right\rangle\left\langle\phi_{a}^{k}\right|
$$

This operator defines a positive-definite inner product $\langle\cdot, \cdot\rangle_{+}:=\left\langle\cdot \mid \eta_{+} \cdot\right\rangle$ that renders $H$ self-adjoint and specifies the physical Hilbert space $\mathcal{H}_{\text {phys }}$ of the model [2]. Note, however, that the metric operator (20) is not unique [28] - [31]; one can use $\phi_{a}^{\prime} k$ of (19) to construct other admissible metric operators. Indeed, the determination of the coefficient functions $N_{a}(z, k)$ that would reproduce the usual metric operator $\left(\eta_{+}=1\right)$ and the $\left(L^{2}-\right)$ inner product in the Hermitian limit $(\Im(z) \rightarrow 0)$ is a very difficult problem. The only guiding principle is to make a simple choice for the biorthonormal

system that shares the symmetries of the Hamiltonian. In the following we will see that the choice (16)-(17) made in the preceding section does indeed fulfill this highly nontrivial requirement.

Having made a choice for $\phi_{a}^{k}$, we can try to compute the integral kernel for $\eta_{+}$, namely

$$
\eta_{+}(\mathrm{x}, \mathrm{y}):=\left\langle\mathrm{x}\left|\eta_{+}\right| \mathrm{y}\right\rangle=\sum_{a=1}^{2} \int_{0}^{\infty} d k \phi_{a}^{k}(\mathrm{x}) \phi_{a}^{k}(\mathrm{y})^{*}
$$


Substituting (16) and (17) in this equation and simplifying the result, we obtain

$$
\begin{aligned}
\eta_{+}(\mathrm{x}, \mathrm{y})= & \frac{1}{2}[\delta(\mathrm{x}-\mathrm{y})-\delta(\mathrm{x}+\mathrm{y})]+\alpha(\mathrm{x}, \mathrm{y})+z \beta(\mathrm{x}, \mathrm{y}) \operatorname{sign}(\mathrm{y})+ \\
& z^{*} \beta(\mathrm{y}, \mathrm{x}) \operatorname{sign}(\mathrm{x})+|z|^{2} \gamma(\mathrm{x}, \mathrm{y}) \operatorname{sign}(\mathrm{x}) \operatorname{sign}(\mathrm{y})
\end{aligned}
$$

where

$$
\begin{aligned}
\alpha(\mathrm{x}, \mathrm{y}) & :=\frac{1}{2 \pi} \int_{-\infty}^{\infty} d k \frac{\cos (k \mathrm{x}) \cos (k \mathrm{y})}{\left|1+\frac{z^{2}}{4 k^{2}}\right|}=\frac{1}{4 \pi}\left[I_{0}(\mathrm{x}+\mathrm{y})+I_{0}(\mathrm{x}-\mathrm{y})\right] \\
\beta(\mathrm{x}, \mathrm{y}) & :=\frac{1}{4 \pi} \int_{-\infty}^{\infty} d k \frac{\cos (k \mathrm{x}) \sin (k \mathrm{y})}{k\left|1+\frac{z^{2}}{4 k^{2}}\right|}=\frac{1}{8 \pi i}\left[I_{1}(\mathrm{x}+\mathrm{y})+I_{1}(\mathrm{y}-\mathrm{x})\right] \\
\gamma(\mathrm{x}, \mathrm{y}) & :=\frac{1}{8 \pi} \int_{-\infty}^{\infty} d k \frac{\sin (k \mathrm{x}) \sin (k \mathrm{y})}{k^{2}\left|1+\frac{z^{2}}{4 k^{2}}\right|}=\frac{1}{16 \pi}\left[I_{2}(\mathrm{x}-\mathrm{y})-I_{2}(\mathrm{x}+\mathrm{y})\right] \\
I_{n}(r) & :=\int_{-\infty}^{\infty} d k \frac{e^{i r k}}{k^{n}\left|1+\frac{z^{2}}{4 k^{2}}\right|}=\int_{-\infty}^{\infty} d k \frac{e^{i r k}}{k^{n} \sqrt{\left(1+\frac{z^{2}}{4 k^{2}}\right)\left(1+\frac{z^{* 2}}{4 k^{2}}\right)}}
\end{aligned}
$$

$r \in \mathbb{R}$, and $n=0,1,2$. As seen from (22) - (26) , the calculation of $\eta_{+}(x, y)$ reduces to that of $I_{n}(r)$. The latter cannot be evaluated in a closed form. We will construct a series expansion for $I_{n}(r)$ that would allow for a perturbative treatment of the problem.

First, we introduce

$$
\begin{aligned}
& a:=\frac{\Re(z)^{2}}{4}, \quad b:=\frac{\Im(z)^{2}}{4}, \quad \epsilon:=\frac{\Im(z)}{\Re(z)}=\sqrt{\frac{b}{a}}, \\
& s:=\frac{\Re(z) r}{2}=\sqrt{a} r, \quad q:=\frac{2 k}{\Re(z)}=\frac{k}{\sqrt{a}},
\end{aligned}
$$

where $\Re$ and $\Im$ stand for the real and imaginary part of their argument, respectively 4

It is not difficult to show that

$$
I_{n}(r)=\left.a^{(1-n) / 2} \int_{-\infty}^{\infty} d q \frac{e^{i s q} q^{2-n} f(q, \epsilon)}{q^{2}+1}\right|_{s=\sqrt{a} r},
$$

where

$$
f(q, \epsilon):=\left(1+\frac{\left[\epsilon^{2}+2\left(1-q^{2}\right)\right] \epsilon^{2}}{\left(q^{2}+1\right)^{2}}\right)^{-1 / 2} .
$$

In view of these equations, we may use $\epsilon$ as an appropriate non-Hermiticity parameter. In the following we will construct a perturbative expansion of the metric operator in terms of $\epsilon$.

Expanding $f(q, \epsilon)$ in powers of $\epsilon$, we have

$$
f(q, \epsilon)=1+\frac{q^{2}-1}{\left(q^{2}+1\right)^{2}} \epsilon^{2}+\frac{q^{4}-4 q^{2}+1}{\left(q^{2}+1\right)^{4}} \epsilon^{4}+\mathcal{O}\left(\epsilon^{6}\right),
$$

\footnotetext{
${ }^{4}$ Note that $\Re(z)$ and $a$ are both positive.
} 
where $\mathcal{O}\left(\epsilon^{N}\right)$ stands for terms of order $N$ and higher in powers of $\epsilon$. Substituting (31) in (29) and evaluating the resulting integrals yields, after a very lengthy calculation that is partly done by Mathematica, the following remarkably simple result.

$$
\begin{aligned}
& I_{0}(r)=\left.2 \pi \sqrt{a}\left\{\delta(s)-\frac{e^{-|s|}}{2}+\left[\frac{e^{-|s|}}{8}\left(s^{2}-3|s|+1\right)\right] \epsilon^{2}\right\}\right|_{s=\sqrt{a} r}+\mathcal{O}\left(\epsilon^{4}\right), \\
& I_{1}(r)=\left.i \pi \operatorname{sign}(s) e^{-|s|}\left\{1+\left[\frac{1}{4}(1-|s|)|s|\right] \epsilon^{2}\right\}\right|_{s=\sqrt{a} r}+\mathcal{O}\left(\epsilon^{4}\right), \\
& I_{2}(r)=\left.\frac{\pi}{\sqrt{a}} e^{-|s|}\left\{1+\left[-\frac{1}{4}\left(s^{2}+|s|+1\right)\right] \epsilon^{2}\right\}\right|_{s=\sqrt{a} r}+\mathcal{O}\left(\epsilon^{4}\right) .
\end{aligned}
$$

Having obtained $I_{n}(r)$, we are in a position to derive an explicit perturbative expansion for the metric operator:

$$
\eta_{+}(\mathrm{x}, \mathrm{y})=\sum_{m=0}^{N-1} \eta_{+}^{(m)}(\mathrm{x}, \mathrm{y}) \epsilon^{m}+\mathcal{O}\left(\epsilon^{N}\right)
$$

where $N=1,2,3, \cdots$ and $\eta_{+}^{(m)}(\mathrm{x}, \mathrm{y})$ is independent of $\epsilon$. Inserting (32) - (34) in (23) - (25), using the resulting expression to write (22) in the form (35), and employing various properties of "sign", particularly

$$
\operatorname{sign}(\mathrm{x}+\mathrm{y})[\operatorname{sign}(\mathrm{x})+\operatorname{sign}(\mathrm{y})]=1+\operatorname{sign}(\mathrm{x}) \operatorname{sign}(\mathrm{y})=2 \theta(\mathrm{xy})
$$

where $\theta(\mathrm{x}):=[1+\operatorname{sign}(\mathrm{x})] / 2$ is the step function, we find after miraculous cancellations of a large number of terms

$$
\begin{aligned}
& \eta_{+}^{(0)}(\mathrm{x}, \mathrm{y})=\delta(\mathrm{x}-\mathrm{y}) \\
& \eta_{+}^{(1)}(\mathrm{x}, \mathrm{y})=\frac{i \Re(z)}{4}\left[\theta(\mathrm{xy}) e^{-\Re(z)|\mathrm{x}-\mathrm{y}| / 2}+\theta(-\mathrm{xy}) e^{-\Re(z)|\mathrm{x}+\mathrm{y}| / 2}\right] \operatorname{sign}\left(\mathrm{y}^{2}-\mathrm{x}^{2}\right), \\
& \eta_{+}^{(2)}(\mathrm{x}, \mathrm{y})= \frac{\Re(z)}{16}\left\{[-\Re(z)|\mathrm{x}-\mathrm{y}| \theta(\mathrm{xy})+\theta(-\mathrm{xy})] e^{-\Re(z)|\mathrm{x}-\mathrm{y}| / 2}+\right. \\
& \eta_{+}^{(3)}(\mathrm{x}, \mathrm{y})= \frac{i \Re(z)^{2}}{32}\left\{\theta(\mathrm{xy})|\mathrm{x}-\mathrm{y}|\left(1-\frac{1}{2} \Re(z)|\mathrm{x}-\mathrm{y}|\right) e^{-\Re(z)|\mathrm{x}-\mathrm{y}| / 2}+\right. \\
&\left.\theta(-\mathrm{xy})|\mathrm{x}+\mathrm{y}|\left(1-\frac{1}{2} \Re(z)|\mathrm{x}+\mathrm{y}|\right) e^{-\Re(z)|\mathrm{x}+\mathrm{y}| / 2}\right\} \operatorname{sign}\left(\mathrm{y}^{2}-\mathrm{x}^{2}\right) .
\end{aligned}
$$

We should emphasize that according to (36) the metric operator $\eta_{+}$tends to the identity operator in the Hermitian limit: $\epsilon \rightarrow 0$. This is by no means a trivial expectation. It is a consequence of our choice for the biorthonormal system. Moreover $\eta_{+}^{(m)}(\mathrm{x}, \mathrm{y})$ satisfy the Hermiticity condition, $\eta_{+}^{(m)}(\mathrm{x}, \mathrm{y})^{*}=\eta_{+}^{(m)}(\mathrm{y}, \mathrm{x})$, manifestly. A more important property of $\eta_{+}^{(m)}(\mathrm{x}, \mathrm{y})$ is that they define bounded (integral) operators $\eta_{+}^{(m)}$ in all of $L^{2}(\mathbb{R})$. This can be established using the fact that the integrals $\int_{-\infty}^{\infty}\left|\eta_{+}^{(m)}(\mathrm{x}, \mathrm{y})\right| d \mathrm{y}$ are bounded for all $x \in \mathbb{R}$, [32, $\S$ III.2.1]. Alternatively, we may employ the following direct proof of the boundedness of $\eta_{+}^{(m)}$ for $m=0,1,2,3$, 333. First we observe that $\left|\eta_{+}^{(m)}(\mathrm{x}, \mathrm{y})\right|$ viewed as a function of $\mathrm{x}$ has an upper bound, $\mu^{(m)}(\mathrm{y})$, depending on $\mathrm{y}$ such that 
$c:=\int_{-\infty}^{\infty} \mu^{(m)}(\mathrm{y}) d \mathrm{y}<\infty$. This implies that for all $\psi \in L^{2}(\mathbb{R})$,

$$
\begin{aligned}
\left\|\eta_{+}^{(m)} \psi\right\|^{2} & =\int_{-\infty}^{\infty} d \mathrm{x}\left|\int_{-\infty}^{\infty} d \mathrm{y} \eta_{+}^{(m)}(\mathrm{x}, \mathrm{y}) \psi(\mathrm{y})\right|^{2} \\
& \leq \int_{-\infty}^{\infty} d \mathrm{x}\left(\int_{-\infty}^{\infty} d \mathrm{y} \sqrt{\mu^{(m)}(\mathrm{x}) \mu^{(m)}(\mathrm{y})}|\psi(\mathrm{y})|\right)^{2} \\
& \leq \int_{-\infty}^{\infty} d \mathrm{x} \mu^{(m)}(\mathrm{x}) \int_{-\infty}^{\infty} d \mathrm{y} \mu^{(m)}(\mathrm{y}) \int_{-\infty}^{\infty} d \mathrm{x}|\psi(\mathrm{y})|^{2}=c^{2}\|\psi\|^{2}
\end{aligned}
$$

where we have used the identities: $\left|\eta_{+}^{(m)}(\mathrm{x}, \mathrm{y})\right| \leq \mu^{(m)}(\mathrm{y}),\left|\eta_{+}^{(m)}(\mathrm{x}, \mathrm{y})\right|=\left|\eta_{+}^{(m)}(\mathrm{y}, \mathrm{x})\right| \leq \mu^{(m)}(\mathrm{x})$, and the Schwarz inequality [34]. This completes the proof of the boundedness of $\eta_{+}^{(m)}$ for $m=0,1,2,3$. It implies that at least up to the third order terms in $\epsilon, \eta_{+}$is a bounded operator acting in all of $L^{2}(\mathbb{R})$.

We conclude this section by noting that although the Hamiltonian $H$ is manifestly non- $\mathcal{P} \mathcal{T}$ symmetric, we can follow the approach pursued in [27] to define an antilinear symmetry generator (generalized $\mathcal{P} \mathcal{T}$-operator [29]) for this Hamiltonian according to

$$
\tau:=\sum_{a=1}^{2} \int_{0}^{\infty} d k(-1)^{a}\left|\psi_{a}^{k}\right\rangle \star\left\langle\phi_{a}^{k}\right|
$$

where $\star$ is complex-conjugation operator defined by $(\star\langle\xi|)|\zeta\rangle:=\langle\zeta \mid \xi\rangle$, for all $\psi, \zeta \in L^{2}(\mathbb{R})$. In view of (16), (17), and (41),

$$
\langle\mathrm{x}|\tau| \mathrm{y}\rangle=\sum_{a=1}^{2} \int_{0}^{\infty} d k(-1)^{a} \psi_{a}^{k}(\mathrm{x}) \phi_{a}^{k}(\mathrm{y})=\sum_{a=1}^{2} \int_{0}^{\infty} d k(-1)^{a} \psi_{a}^{k}(\mathrm{x}) \psi_{a}^{k}(\mathrm{y})^{*} .
$$

We will not attempt to obtain a more explicit expression for $\tau$, because unlike the metric operator $\tau$ does not enter in the calculation of the physically relevant quantities.

\section{Equivalent Hermitian Hamiltonian}

Having obtained a perturbative expansion for the metric operator we may proceed with the calculation of the equivalent Hermitian Hamiltonian [9, 2, 4, 5, 35] - 39]:

$$
h:=\eta_{+}^{1 / 2} H \eta_{+}^{-1 / 2}
$$

Using the exponential representation of the metric operator [40]: $\eta_{+}=e^{-Q}$ with $Q=\sum_{m=1}^{\infty} Q_{m} \epsilon^{m}$, the pseudo-Hermiticity relation [12]: $H^{\dagger}=\eta_{+} H \eta_{+}^{-1}$, and the fact that $Q_{1}=-\eta^{(1)}$, we first calculate the equivalent Hermitian Hamiltonian corresponding to the dimensionless Hamiltonian $\mathrm{H}$. The result is 30.

$$
\mathrm{h}=-\frac{d^{2}}{d \mathrm{x}^{2}}+\Re(z) \delta(\mathrm{x})+\mathrm{h}^{(2)} \epsilon^{2}+\mathcal{O}\left(\epsilon^{3}\right)
$$


where

$$
\mathrm{h}^{(2)}:=\frac{i \Re(z)}{4}\left[\eta_{+}^{(1)}, \delta(\mathrm{x})\right] .
$$

In view of (37) and (44), we can easily compute

$$
\begin{aligned}
\left\langle\mathrm{x}\left|\mathrm{h}^{(2)}\right| \mathrm{y}\right\rangle & =\frac{i \Re(z)}{4}[\delta(\mathrm{y})-\delta(\mathrm{x})] \eta_{+}^{(1)}(\mathrm{x}, \mathrm{y}) \\
& =\frac{\Re(z)^{2}}{16}\left[\delta(\mathrm{x}) e^{-\Re(z)|\mathrm{y}| / 2}+\delta(\mathrm{y}) e^{-\Re(z)|\mathrm{x}| / 2}\right],
\end{aligned}
$$

where the latter expression is to be treated in the sense of distributions (it is valid inside an integral).

Next, we express the Hermitian Hamiltonian $h$ in terms of the original (unscaled) physical variables and the relevant length scale of the problem which, as we explain below, is given by

$$
L:=\frac{\hbar^{2}}{m \Re(\zeta)} .
$$

This yields

$$
h=\frac{p^{2}}{2 m}+\Re(\zeta) \delta(x)+\Im(\zeta)^{2} h^{(2)}+\mathcal{O}\left(\Im(\zeta)^{3}\right),
$$

where $h^{(2)}$ is defined in terms of its integral kernel,

$$
h^{(2)}(x, y):=\frac{m}{8 \hbar^{2}}\left[\delta(x) e^{-|y| / L}+\delta(y) e^{-|x| / L}\right],
$$

according to

$$
\begin{aligned}
\left(h^{(2)} \psi\right)(x) & :=\int_{-\infty}^{\infty} h^{(2)}(x, y) \psi(y) d y=c_{0}[\psi] e^{-|x| / L}+c_{1}[\psi] \delta(x), \\
c_{0}[\psi] & :=\frac{m \psi(0)}{8 \hbar^{2}}, \quad c_{1}[\psi]:=\frac{m}{8 \hbar^{2}} \int_{-\infty}^{\infty} e^{-|y| / L} \psi(y) d y .
\end{aligned}
$$

Clearly $h^{(2)}$ and consequently $h$ are nonlocal operators [2]. Furthermore, they are both real $(\mathcal{T}$ symmetric) and $\mathcal{P}$-symmetric 5

To demonstrate the physical consequences of the imaginary part of the coupling constant $\zeta$ in the original Hamiltonian (1) and appreciate the meaning of the length scale $L$, we calculate the energy expectation value for a Gaussian position wave function centered at $x=0$ and having mean momentum $\bar{p}=\hbar k$ and width $\sigma$,

$$
\Psi(x)=\left(\pi \sigma^{2}\right)^{-1 / 4} e^{-\frac{x^{2}}{2 \sigma^{2}}+i k x} .
$$

It is important to note that we work solely in the Hermitian representation of the quantum system where $h$ represents the Hamiltonian of the system and $x$ the position operator. Naturally, we view $\Psi$ as an element of $\mathcal{H}$ which yields the probability density of the localization of the particle in the physical space as $|\Psi(x)|^{2}$. The corresponding element of $\mathcal{H}_{\text {phys }}$ is given by $\psi=\eta_{+}^{-1 / 2} \Psi$, [2].

\footnotetext{
${ }^{5}$ Another property of $h^{(2)}$ is that it eliminates odd wave functions.
} 


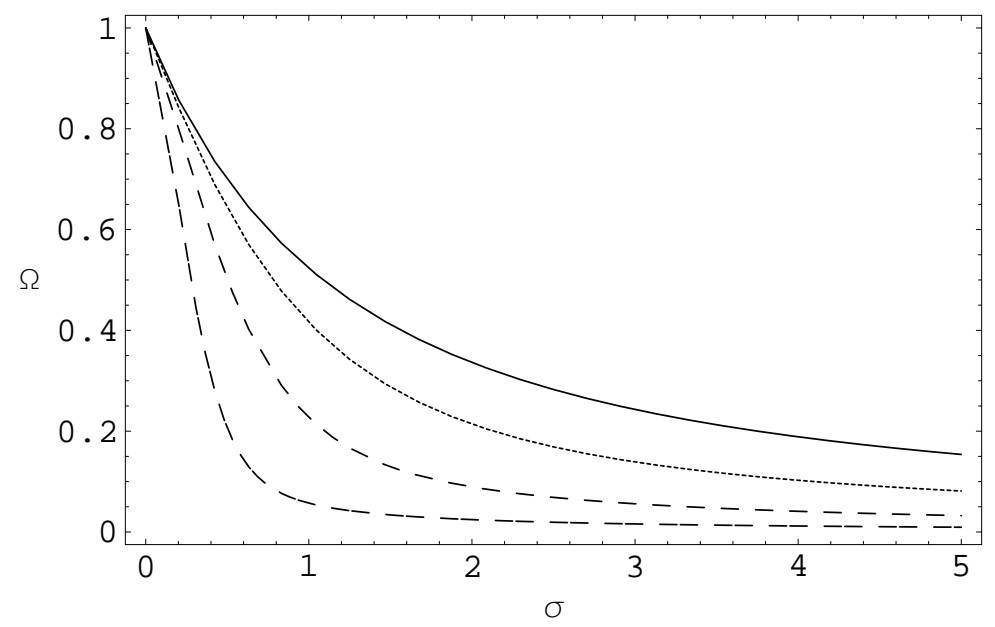

Figure 1: Plots of $\Omega$ as a function of $\sigma$ for $k=0$ (the full curve), $k=1$ (the dotted curve), $k=2$ (the dashed curve), and $k=4$ (the dashed-dotted curve) in units where $L=1$.

The energy expectation value of a particle in the state described by the (normalized) position wave function (50) has the form

$$
\langle\Psi|h| \Psi\rangle=\frac{\hbar^{2}\left(\sigma^{-2}+2 k^{2}\right)}{4 m}+\frac{\Re(\zeta)}{\sqrt{\pi} \sigma}+\left(\frac{m \Omega(\sigma, k)}{2^{3 / 2} \hbar^{2}}\right) \Im(\zeta)^{2}+\mathcal{O}\left(\Im(\zeta)^{3}\right),
$$

where

$$
\Omega(\sigma, k):=e^{-\frac{1}{2}\left(k^{2}-L^{-2}\right) \sigma^{2}}\left[\cos \left(L^{-1} k \sigma^{2}\right)-\Re\left\{e^{i L^{-1} k \sigma^{2}} \operatorname{erf}\left[2^{-1 / 2}\left(L^{-1}+i k\right) \sigma\right]\right\}\right],
$$

and $\operatorname{erf}(x):=2 \pi^{-1 / 2} \int_{0}^{x} e^{-y^{2}} d y$ is the error function. The presence of the exponential factor on the right-hand side of (52) suggests that the non-Hermiticity effect decays rapidly for mean momentum values $\bar{p}=\hbar k$ outside the range $\left[-\hbar L^{-1}, \hbar L^{-1}\right]$. Figure 1 shows the plots of $\Omega$ as a function of $\sigma$ for various values of $k$. As one increases $|k|$ the non-Hermiticity effect diminishes. The maximum is attained for a stationary Gaussian wave packet $(k=0)$ for which

$$
\langle\Psi|h| \Psi\rangle=\frac{\hbar^{2}}{4 m \sigma^{2}}+\frac{\Re(\zeta)}{\sqrt{\pi} \sigma}+\left(\frac{m e^{\frac{\sigma^{2}}{2 L^{2}}}\left[1-\operatorname{erf}\left(2^{-1 / 2} L^{-1} \sigma\right)\right]}{2^{3 / 2} \hbar^{2}}\right) \Im(\zeta)^{2}+\mathcal{O}\left(\Im(\zeta)^{3}\right) .
$$

It is not difficult to show that for such a wave packet

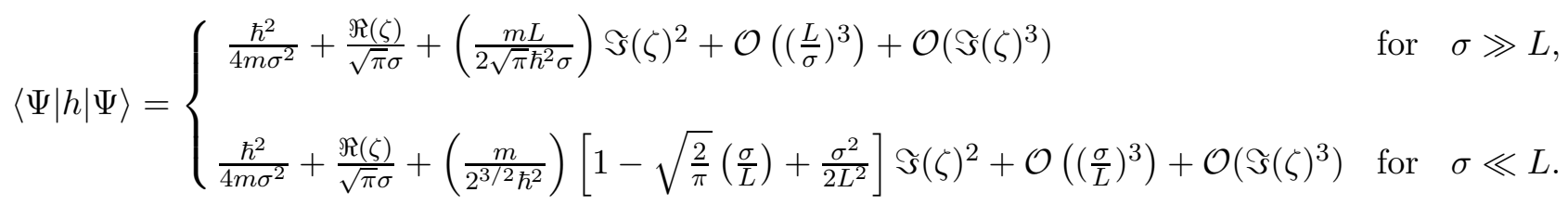

Next, we compute the energy expectation value for a stationary Gaussian wave packet of width $\sigma$ and mean position $a$,

$$
\Psi(x)=\left(\pi \sigma^{2}\right)^{-1 / 4} e^{-\frac{(x-a)^{2}}{2 \sigma^{2}}} .
$$




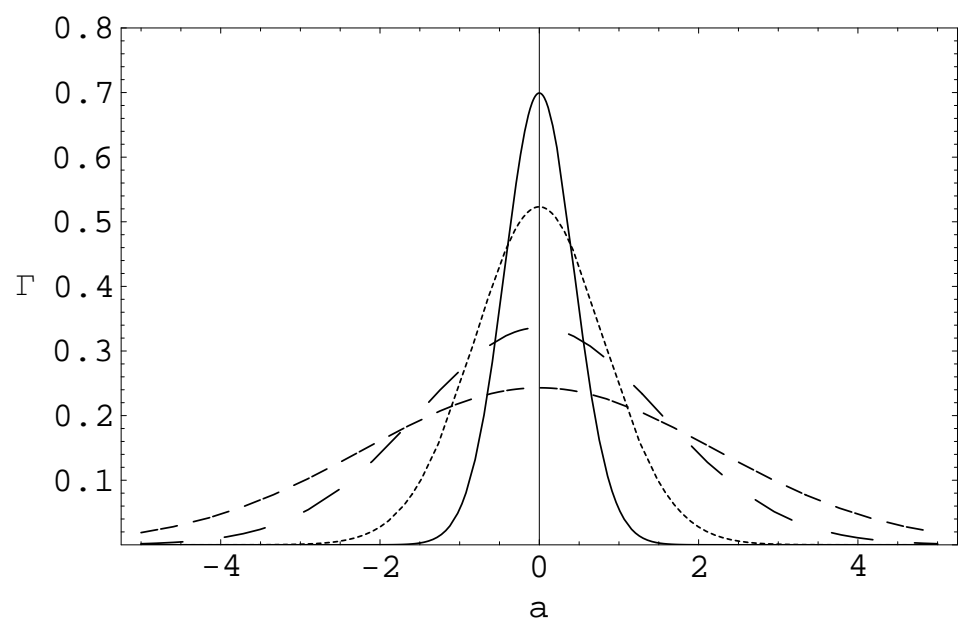

Figure 2: Plots of $\Gamma$ as a function of $a$ for $\sigma=0.5$ (the full curve), $\sigma=1$ (the dotted curve), $\sigma=2$ (the dashed curve), and $\sigma=3$ (the dashed-dotted curve) in units where $L=1$.

The result is

$$
\langle\Psi|h| \Psi\rangle=\frac{\hbar^{2}}{4 m \sigma^{2}}+\frac{e^{-\frac{a^{2}}{2 L^{2}} \Re(\zeta)}}{\sqrt{\pi} \sigma}+\left(\frac{m \Gamma(\sigma, a)}{2^{3 / 2} \hbar^{2}}\right) \Im(\zeta)^{2}+\mathcal{O}\left(\Im(\zeta)^{3}\right)
$$

where

$$
\Gamma(\sigma, a):=e^{-\frac{1}{2}\left(\frac{a^{2}}{\sigma^{2}}-\frac{\sigma^{2}}{L^{2}}\right)}\left\{\cosh \left(\frac{a}{L}\right)-\frac{1}{2} e^{\frac{a}{L}} \operatorname{erf}\left[2^{-1 / 2}\left(\frac{\sigma}{L}+\frac{a}{\sigma}\right)\right]-\frac{1}{2} e^{-\frac{a}{L}} \operatorname{erf}\left[2^{-1 / 2}\left(\frac{\sigma}{L}-\frac{a}{\sigma}\right)\right]\right\} .
$$

Figure 2 shows the plots of $\Gamma$ as a function of $a$ for various values of $\sigma$. As seen from these plots the non-Hermiticity effect is substantially smaller for mean positions outside $[-L, L]$. Therefore, $L$ determines the range of the non-Hermitian (nonlocal) interaction.

In order to make a crude estimate for the magnitude $L$, consider the application of the delta function potential in modelling a point defect in a one-dimensional electron gas system. If we take the spatial size $d$ of the defect (lattice size of the crystal) to be of the order of 1 Angstrom and the strength of the real part of the potential 6 to be of the order of $1 \mathrm{ev}$, for an electron (of usual mass) we find $L$ to be of the order $10^{-10}$ Angstrom! Similarly we can obtain an order of magnitude estimate for the strength of the non-Hermitian interaction namely $m \Re(z)^{2} /\left(8 \hbar^{2} d\right)$. This turns out to be $10^{8} \epsilon^{2}$ ev. Therefore, to ensure the validity of our perturbative calculation of $h$, we need to take $\epsilon \ll 10^{-4}$. We also recall that the non-Hermitian interaction will be significant, if it is stronger than the thermal effects, i.e., $m \Re(z)^{2} /\left(8 \hbar^{2} d\right)>k T$. At room temperature $\left(k T \approx 10^{-2} \mathrm{ev}\right)$, this implies $\epsilon>10^{-5}$.

Finally, we would like to point out that the calculation of the energy expectation value can be performed using the pseudo-Hermitian representation of the system. This requires calculation of the state vector $\psi=\eta_{+}^{-1 / 2} \Psi$ corresponding to the position wave function $\Psi$. The energy

\footnotetext{
${ }^{6}$ Here we write the real part of the potential as $d^{-1} \Re(z) \delta\left(d^{-1} x\right)$ and identify $d^{-1} \Re(z)$ with its strength.
} 
expectation value then takes the form $\langle\psi, H \psi\rangle_{+}=\left\langle\psi \mid \eta_{+} H \psi\right\rangle$. This calculation is by no means easier to perform than the one reported above [11, 41]. It simplifies to some extend, if one chooses $\psi$ directly, e.g., identify $\psi(x)$ with a Gaussian wave packet. However, note that $\psi(x)$ is void of a direct physical meaning; it is not the position wave function for the state it describes. In order to assign a physical meaning for $\psi$ in terms of the position of the particle, one must compute the corresponding position wave function [2], namely $\Psi=\eta_{+}^{1 / 2} \psi$.

\section{Conclusion}

In this paper we applied the machinery of pseudo-Hermitian quantum mechanics to explore a unitary quantum system determined by a delta function potential with a complex coupling constant $\zeta$. For an imaginary coupling constant there exists a spectral singularity. For $\Re(\zeta)>0$ the spectrum is purely continuous and one can construct a complete biorthonormal system. The double degeneracy of the spectrum complicates the choice of a biorthonormal system. We selected an appropriate biorthonormal system that simplified the calculations and had a symmetric expression for the pair of basis eigenfunctions associated with each degeneracy subspace. We then constructed the corresponding metric operator $\eta_{+}$perturbatively and showed that it tended to the identity operator in the non-Hermitian limit and was indeed a bounded operator at least up to and including the third order terms that we computed. This is quite remarkable, for there are an infinity of other biorthonormal systems such that the corresponding metric operator is either unbounded or fails to yield the identity operator in the Hermitian limit.

Next, we constructed the equivalent nonlocal Hermitian Hamiltonian $h$ for the system. The nature of the nonlocality of $h$ is quite intriguing, because it originates from a complex delta-function potential which is actually ultra-local! This seems to be the reason why the non-Hermiticity effect appears in the Hermitian Hamiltonian in the form of a short range interaction, i.e. it decays rapidly outside the interaction region: $[-L, L]$, where $L=\frac{\hbar^{2}}{m \Re(\zeta)}$. To establish this we calculated the expectation value of energy for various Gaussian position wave functions. For a non-stationary Gaussian wave packet centered at the origin, the non-Hermitian effect reaches its maximum for mean momenta in the range $\left[-\hbar L^{-1}, \hbar L^{-1}\right]$. For a stationary wave packet, it becomes sizable whenever the mean position of the packet lies within the interaction region $[-L, L]$.

The results reported above show how the methods developed for treating systems with a discrete spectrum [7, 2] generalize to specific models with a continuous spectrum. Such a generalization has previously been employed in the treatment of the $\mathcal{P} \mathcal{T}$-symmetric potential (3) as reported in [5]. The delta function potential considered in the present paper is manifestly non- $\mathcal{P} \mathcal{T}$-symmetric, yet we could successfully apply the methods of pseudo-Hermitian quantum mechanics [2, 3, 4, 30] to reveal its physical content. 


\section{Acknowledgment}

During the course of this work I have benefitted from helpful discussions with Alkan Kabakçığlu and Varga Kalantarov.

\section{References}

[1] C. M. Bender and S. Boettcher, Phys. Rev. Lett. 80, 5243 (1998).

[2] A. Mostafazadeh and A. Batal, J. Phys. A 37, 11645 (2004).

[3] A. Mostafazadeh, J. Phys. A 38, 3213 (2005).

[4] A. Mostafazadeh, J. Phys. A 38, 6557 and 8185 (2005).

[5] A. Mostafazadeh, J. Math. Phys. 46, 102108 (2005).

[6] A. Mostafazadeh, quant-ph/0606173, Czech J. Phys., to appear.

[7] A. Mostafazadeh, J. Math. Phys. 43, 2814 and 3944 (2002).

[8] L. Solombrino, J. Math. Phys. 43, 5439 (2002).

[9] A. Mostafazadeh, J. Phys. A 36, 7081 (2003).

[10] A. Mostafazadeh, quant-ph/0310164

[11] A. Mostafazadeh, Czech J. Phys. 54, 1125 (2004).

[12] A. Mostafazadeh, J. Math. Phys. 43, 205 (2002).

[13] M. A. Naimark, Amer. Math. Soc. Transl. (2), 16, 103 (1960).

[14] H. F. Jones, Phys. Lett. A 262, 242 (1999).

[15] Z. Ahmed, Phys. Lett. A 286, 231 (2001).

[16] S. Albeverio, A.-M. Fei, and P. Kurasov, Lett. Math. Phys. 59, 227 (2002).

[17] R. N. Deb, A. Khare and B. D. Roy, Phys. Lett. A 307, 215 (2003).

[18] E. Demiralp, Czech. J. Phys. 55, 1081 (2005)

[19] H. Uncu and E. Demiralp, "Bound state solutions of the Schrödinger equation for a $\mathcal{P} \mathcal{T}$-symmetric potential with Dirac delta functions," to appear in Phys. Lett. A.

[20] M. Znojil and Vit Jakubski, J. Phys. A 385041 (2005); ibid Czech. J. Phys. 551113 (2005)

[21] A. Mostafazadeh, J. Math. Phys. 47, 072103 (2006).

[22] B. F. Samsonov, J. Phys. A 38, L571 (2005). 
[23] U. V. Riss and H.-D. Meyer, J. Phys. A 28, 1475 (1995).

[24] Z. Ahmed, C. M. Bender, and M. V. Berry, J. Phys. A 38, L627 (2005).

[25] Z. Ahmed, J. Phys. A 39, 7341 (2006).

[26] A. Mostafazadeh, Class. Quantum Grav. 20, 155 (2003).

[27] A. Mostafazadeh, Int. J. Mod. Phys. A 21, 2553 (2006).

[28] F. G. Scholtz, H. B. Geyer, and F. J. W. Hahne, Ann. Phys. (NY) 21374 (1992).

[29] A. Mostafazadeh, J. Math. Phys. 44, 974 (2003).

[30] A. Mostafazadeh, J. Phys. A. 39, 10171 (2006).

[31] F. G. Scholtz, H. B. Geyer, Phys. Lett. B 634, 84 (2006).

[32] T. Kato, Perturbation Theory for Linear Operators, Springer, Berlin, 1995.

[33] V. Kalantarov, private communications.

[34] M. Reed and B. Simon, Functional Analysis, vol. I, Academic Press, San Diego, 1980.

[35] H. F. Jones, J. Phys. A 38, 1741 (2005).

[36] A. Banerjee, Mod. Phys. Lett. A 20, 3013 (2005).

[37] H. F. Jones and J. Mateo, Phys. Rev. D 73085002 (2006).

[38] B. Bagchi, C. Quesne, and R. Roychoudhury, J. Phys. A 39, L127 (2006).

[39] C. Figueira de Morisson and A. Fring, J. Phys. A 39, 9269 (2006).

[40] C. M. Bender, D. C. Brody and H. F. Jones, Phys. Rev. D 70, 025001 (2004).

[41] A. Mostafazadeh, hep-th/0603059. 